\title{
Criminology Tracking Project •
}

\section{Why \& How to Be a CTP Tagger}

Published on: Sep 26, 2021

DOI: $10.21428 / \mathrm{ad} 528678 . e 15 \mathrm{aff} 60$

License: Creative Commons Attribution 4.0 International License (CC-BY 4.0), 


\section{Introduction}

When you tag for CTP, you push useful information to our feed subscribers and store the records in the CTP database for deduping, export, preservation, updating, and search. 1 This helps the criminology community in several concrete ways that foster the field:

- It spreads the word about new developments.

- It supports criminology research and teaching.

- It supports easy sharing of comprehensive, current, always-growing collections of criminology's W5H. $\underline{2}$

\section{We Welcome Taggers of All Kinds}

\section{Generalists and Specialists}

Some taggers are wide ranging and tag anything about criminology. Others focus on a given aspect of W5H or their respective subtypes, even if they also tag outside that area. For example, they may focus on particular types of people (e.g., researchers), outputs (e.g., articles, videos), places (e.g., journals or departments), or branches (e.g., theories and methods), but still tag outside that area when they see something to share.

\section{Gatherers and Searchers}

Some people only tag what they happen to notice, adding a light layer of tagging to their ordinary routine. Others search systematically for criminology information, at least in a given niche. For example, if you are committed to tagging all criminology activity in a certain subtype, you might start with a systematic search for past activity therein. Or every few days, you might systematically search for recent additions and tag them. If you are researching or teaching (an aspect of) criminology, then you are already doing a systematic search for relevant information and, thus, only need to make sure it is also tagged for CTP.

\section{Hounds and Historians}

Some taggers focus on new developments because they are trying to keep up. They use CTP to share what they discover. Others focus on older developments because they are researching or teaching (an aspect of) criminology and want to make the relevant CTP tags retroactively comprehensive. 


\section{Regulars and Irregulars}

Some people tag every day or several days a week. Others tag sporadically. Every little bit helps.

\section{Recommenders}

Some individual and groups will create what we call a recommendation feed by tagging the items they would like to highlight as most worth attention.

Recommendation feeds can be on criminology in general or an aspect of it.

\section{Persuaded?}

We hope you will give tagging a try. Below is how to get started as a tagger.

\section{How to Get Started as a Tagger}

\section{Get a TagTeam Account}

1. Go to the Harvard instance of TagTeam and click the "Sign in" link in the upper right corner. Or go directly to the "Sign up" page.

2. Fill out the form.

- Note that the Harvard instance of TagTeam is limited to academic or research projects. Use the form to give a brief description of your project.

- If you don't describe an academic or research project, your request for an account may be denied.

- Tagging for CTP definitely counts as an academic or research project. Hence, if you want to tag for CTP, just say that.

- Click the green "Sign up" button (not the blue "Log in" button).

Once you have an account in TagTeam, you may create new hubs (projects), tag for those hubs, and authorize others to tag for them as well. To tag for an existing hub, like CTP, you'll need the permission of the hub owner (see below). If your purpose is to tag for CTP, then please do not create a new criminology-specific hub. Use the CTP hub for tagging all such items.

\section{Get Permission to Tag for CTP}

1. Log in to TagTeam, and go to the CTP hub.

2. Click on the Contact tab in the left sidebar. Fill in the form, for example, by asking for permission to tag for CTP. Click "Submit." 


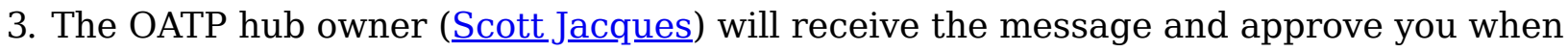
it arrives.

When you get this far, you will be authorized to tag for CTP. The next few sections are about how to do that.

\section{Add and Use the Tagging Bookmarklet}

1. Log in to TagTeam, and go to the CTP hub.

2. Click on the "Taggers" tab in the left sidebar.

3. Click or drag the green "Add to TagTeam" button to your browser's toolbar. (You will only see this button if you're logged in to TagTeam and authorized to tag for CTP.)

4. Surf the web as usual. When you are viewing a web page you want to tag, click on the bookmarklet and fill in the pop-up form.

In a nutshell: When viewing a page you would like to tag, click on the bookmarklet and fill in the form with relevant tags and a short excerpt or summary.

\section{Learn About CTP Tags}

\section{Must Know}

- Use the primary tag (crim.new) for items that are new within the last six months, and omit it otherwise. If you do not know the date of an item, omit the crim.new tag; do not guess.

- Use relevant secondary or subtype tags. Basically, all tags other than crim.new. On the importance of secondary tags, see the section on them in our tagging_guidelines.

- Use descriptions. These are paraphrases or quoted excerpts that help our readers see how the work is relevant to criminology and decide whether to click through to the full text. For more, see the section on them in our tagging_guidelines.

If you are re just getting started then you can skip everything below this point. We include the extra details and suggestions below to help taggers who want to move the next level. Use them as needed, and don't let them intimidate!

\section{Should Know, Eventually}

- Read the FAQ.

- Bookmark the collection of items you tagged. This will help you review and revise your work (see below). 
- Pay attention to the email feedback you automatically receive when other members of CTP add tags or make updates to your tag records. (To scale to a large number of users, this is our primary way of giving taggers feedback. However, if you have questions or would like more personalized feedback, please just let us know.)

- Consult our steadily growing list of major project tags. Know that it is here as a reference.

- Review the most frequently-used tags.

- See how other CTP taggers are tagging.

- Read the CTP tagging_guidelines.

- Review the CTP tag filters, converting some deprecated tags to approved tags.

- Review the section of the TagTeam manual on tagging. The page is for the Open Access Tracking Project (OATP) but equally applicable to CTP. For more background, see the TagTeam home page and TagTeam FAQ.

\section{Revise Your Work}

After you have tagged an item for CTP, you may want to add new tags, or modify or remove existing tags. You may also want revise and improve the work of other taggers. Here is how to do so:

1. Log in to TagTeam, and go to the CTP hub.

2. Go to the tag record for the item you want to update or delete. $\underline{3}$

- To remove an item entirely, look for the gear icon next to the title of each item on the list. Click on that and you'll get a pop-up menu. One of the options is to remove that item from your collection.

- To revise an item ...

3. Click on the "Filters" tab in the left sidebar.

4. Chose one of the three options (add, remove, modify a tag), and fill in the form. $\underline{4}$

Another way to add, remove, or modify tags is to return to the original page on the web, and click on the bookmarklet as if you were going to tag it again. This method also works if you want to modify the title or description. The tagging form will pop up prepopulated with the existing tags, title, URL, and description. Add new tags in the regular way. Delete a tag by clicking the gray " $x$ " to its right. Modify a tag by clicking on it and picking your option from the pop-up menu.

\section{Recommended Practices}

Once you've started as a tagger, consider taking additional steps to help the project. 
- Review your work and revise it as needed (see above).

- Subscribe to the CTP primary feed as a reader. This will show you what your tagging (and the tagging of your peers) looks like to our subscribers.

Most users who subscribe to an unabridged version of CTP primary feed prefer the email version, though the feed comes in many other formats.

(The Twitter version is abridged, against our will.) You might also want to subscribe to one or more secondary CTP feeds.

- Bookmark Google Translate, Microsoft Translate, or DeepL. Even though CTP aims to cover OA-related news in all countries and languages, it aims to do so in English.

- Review the CTP primary feed (all new items) or full feed (all items old and new) from within TagTeam. When you see an item that was inadequately tagged, add some of the missing tags. If you click through to the full tag record and believe that the item was inadequately described, improve the description. If most of your CTP work is on a given subtype, then review and improve the item records within your area(s) of focus.

- Try one or more of the ways to use CTP for your research or teaching OA, and recommend these to your colleagues, students, et cetera.

- Recruit new CTP taggers. When you find promising prospects, share this page on how to get started. Help them learn the basics. CTP is a crowd-sourced project. The more taggers we have, the more comprehensively we can cover criminology. We depend on people like you and those you recruit.

\section{Tips and Suggestions}

- If you click on the TagTeam bookmarklet, and the form pops up already filled in, that means that the page you are viewing has already been tagged for CTP. You could back out and move on, or you could review the tags already applied and think about whether the previous tagger(s) omitted any that you could add now.

- The CTP tagging_guidelines and list of approved tags are both fairly long and might be intimidating. But don't be intimidated. There is a learning curve, but it is not steep.

- Once you start tagging, you will receive automated email feedback when other CTP taggers modify or improve on your work. In this way, all our taggers help one another.

- If you or your organization would like to be systematic in finding and tagging new items of a certain kind, let us know. CTP is trying to recruit taggers to take responsibility-alone or jointly_for items on a certain aspect of criminology. If we get a critical mass of them then we will create a public web page listing them, partly 
to give public thanks and partly to show the areas where we still need dedicated taggers.

- Consider creating a recommendation feed: a feed of items that you would personally like to highlight or recommend. If you are an expert on a certain aspect of criminology, this can be very useful to readers who would like to follow your judgment on new developments in that area.

- You might create your own hub on TagTeam, either to play with the software or to track (academic or research) on topics unrelated CTP. If you do, then you will have tagging rights in more than one hub-CTP plus your own. When you tag a new item, the bookmarklet lets you choose which hub it will go to (in a pull-down menu in the upper left corner). By default new items go to the hub for which you most recently tagged.

- Please only tag items that are on-topic. This is true for all TagTeam hubs. If you tag too many items unrelated to the hub topic, the hub owner may rescind your permission to tag for the hub. TagTeam wants to help researchers who create hubs to publish carefully curated feeds relevant to their topics.

- Note that when you tag for a TagTeam hub, the hub owner and designated other hub participants may change your tags. This is a feature, not a bug. In fact, it is the one feature that most inspired the the creation of TagTeam, given that many platforms already existed to support basic tagging. This feature enables TagTeam projects, like $\mathrm{CTP}$, to manage the evolution of a folksonomy to an ontology, or to convert uncoordinated user-defined tags to a standard vocabulary of project-approved tags.

\section{Footnotes}

1. This page is reused and adapted from https://cyber.harvard.edu/hoap/Why tag_for OATP, https://cyber.harvard.edu/hoap/OATP all-volunteer_phase, and https://cyber.harvard.edu/hoap/Get started as a tagger (as of 9/22/2021).

2. "W5H" stands for who, what, when, where, why, and how. $\bullet$

3. For example, run a search or scroll through the list of items until you find the one you want to update. Then click on the link in the item's title. That takes you to the tag record for that item.

4. These are called item-level filters. Note that permission to tag for a hub doesn't automatically include permission to create item-level filters. You probably have both kinds of permission. But if you don't, then ask the hub owner. 